\title{
中国与COP15一一负责任环境大国的路径选择
}

\author{
邹玥屿 傅钰琳 杨礼荣 万夏林 王 也 刘纪新* \\ (环境保护部环境保护对外合作中心, 北京 100035)
}

\begin{abstract}
摘要: 《生物多样性公约》第15届缔约方大会(COP15)将于2020年在中国召开, 这是《生物多样性公约》国际进 程中一个承上启下的关键节点。本文尝试在国家自身发展阶段和履约动态的历史背景下，以国家环境外交战略为 尺度, 分析东道国承办大会的内在动因与收益。案例表明, 举办较为成功的几次缔约方大会都体现出了与国家总 体外交战略的紧密联系, 取得了与本国国内或所在区域的生态环保政策相呼应的历史性成果, 从而在推动《公约》 进程的同时提振国内生物多样性工作, 实现了边际效益的最大化。中国同样可以借助承办COP15的机会, 逐步实 现生态环境外交由被动到主动、由内向到外向的转型, 与世界共谋全球生态文明建设之路。为实现该目标, 会议 筹备过程中应注重将其列入国家大外交日程，以主动、开放的姿态与国际社会合作，加强与区域及发展中国家的 战略协同, 充分总结 《公约》履约的中国经验、中国方案, 为推动建立更加公正、合理、高效的全球生物多样性 治理体系作出贡献。
\end{abstract}

关键词：生物多样性公约；缔约方大会；环境外交

\section{China and COP 15: a path for responsible environmental power}

Yueyu Zou, Yulin Fu, Lirong Yang, Xialin Wan, Ye Wang, Jixin Liu*

Foreign Economic Cooperation Office, Ministry of Environment Protection, Beijing 100035

\begin{abstract}
The Fifteenth Meeting of the Conference of the Parties to the Convention on Biological Diversity (COP15) will be hosted by China in 2020 and could become a milestone in the history of the Convention. This article aims to identify lessons that can be learnt by China in preparation for COP15. The internal motivations and political gains of several host countries with respect to previous COPs were analyzed by looking at national environmental foreign policies against the backdrop of the country's development and corresponding progress made in convention implementation. This case study of successful COPs indicates that host countries do not treat it as an isolated event but an action under the country's foreign policy strategy, which provides a strong momentum for the country to contribute to the process. Additionally, by formulating host country initiatives in harmony with existing national and regional policies in the field, the host country was able to optimize marginal effects and gains at both the national and global level. China could also make use the opportunity of hosting COP15 to gradually transform its passive and inward-looking eco-environmental foreign policy into an outward-looking one featuring active engagement and work on eco-civilization along with the international community. In preparation for COP15, China should work together with international stakeholders, reinforce regional strategic coordination and synergism with developing countries, and share Chinese experiences in biodiversity conservation in order to contribute to the creation of a fair, rational, and efficient system of global biodiversity governance.
\end{abstract}

Key words: Convention on Biological Diversity; Conference of Parties; environmental foreign policy

20世纪90年代后期，中国作为负责任大国概念 被提出，并迅速为国际社会所知。较为著名的是
1998年泰国总理川・立派对中国应对亚洲金融危机 的评价和2005年美国副国务卿佐利克对华政策演

收稿日期: 2017-09-10; 接受日期: 2017-09-19

* 通讯作者 Author for correspondence. E-mail: liu.jixin@mepfeco.org.cn 
讲中“负责任利益做关方” (responsible stakeholder) 的有关阐述 ${ }^{(1)}$ 。此后, “负责任大国”逐渐成为中国在 对外交往中身份建构的一个重要元素(李宝俊和徐 正源, 2006), 其关注内容也实现了从政治、军事到 经济, 再到生态等非传统安全的演进(秦亚青, 2003)。2015年前后, 中国与美国共同发布《中美气 候变化联合声明》, 在巴黎气候变化大会上与法国、 美国等携手促成《巴黎协定》，树立了中国深度参 与全球环境治理的里程碑, 显示出中国已将生态保 护视为与经济和安全具有同等重要性的全球性挑 战，并尽力在其中有所作为(时殷弘, 2011)。

进入 21 世纪的最初十年, 中国成为了世界第二 大经济体和全球第一碳排放大国。从被动应对到主 动担当, 中国面对伴随发展而来的资源和环境压力 做出了高效、务实的努力, 获得了国际社会的普遍 赞许(张海滨, 2008)。然而, 中国的环境外交具有明 显内向型特征, 更为关注国内问题, 对除气候变化 等少数几个领域之外的国际环境事务参与仍然有 限(于宏源, 2013年)。随着生态文明理论的提出和逐 渐成熟，2015年中共中央、国务院印发的《生态文 明体制改革总体方案》中, 明确提出了“积极参与全 球治理”的方向。2016年, 中国成功申办2020年《生 物多样性公约》 (以下简称《公约》) 第15次缔约方 大会(Conference of Parties, COP)。作为东道国举办 这次在生物多样性领域具有重要节点意义的大会, 既体现了国际社会对中国取得的生物多样性保护 成效的认可, 也显示了中国全面承担起自身在生态 环境问题上的国际责任的信心与决心。

\section{1 《公约》与缔约方大会}

\section{1 《公约》的地位}

《公约》以保护生物多样性、持续利用其组成 部分, 以及公平合理地分享由利用遗传资源而产生 的惠益为三大目标, 于1993年12月29日正式生效, 与《联合国气候变化框架公约》、《联合国防治荒漠 化公约》并称为 “里约三公约”。《公约》下还制定了 《卡塔赫纳生物安全议定书》 (以下简称《生物安全 议定书》) 和《关于获取遗传资源和公正公平分享其 利用所产生惠益的名古屋议定书》(以下简称《名古

(1) 成元生 (1998) 李岗清同泰国总理川·立派会见, 双方强 调进一步发展两国的友好合作关系. 人民日报, 1998 年 12 月 5 日第 1 版.
屋议定书》)。如今 《公约》是签署最为广泛的国际 条约之一, 主权国家中只有美国和梵蒂冈尚未加入, 彰显了国际社会对于生物多样性的广泛共识。《公 约》的重要地位体现在以下两个方面:

(1)生物多样性已成为继气候变化之后又一全 球环境热点。生物多样性为人类生存、社会经济可 持续发展提供公共产品, 是国家粮食安全、生态安 全和人民身心健康的基本保障。作为国家战略资源 的地位日益凸显, 使得生物多样性成为继气候变化 后全球环境治理又一关注热点和博弯焦点。联合国 大会把2011-2020年确定为“联合国生物多样性十 年”, 其“2015后发展议程”制定的17个可持续发展目 标中, “保护、恢复和促进可持续利用陆地生态系统, 可持续地管理森林, 防治荒漠化, 制止和扭转土地 退化, 阻止生物多样性的丧失” (目标15)作为一项 独立目标位列其中(United Nations, 2015)。

(2)《公约》是国际环境公约中的主流公约。作 为全球生物多样性和环境领域的引领性文件, 《公 约》的目标受到了其他环境公约的普遍认可, 在联 合国环境与发展进程中发挥了重大影响力。多家联 合国机构如开发计划署(UNDP)和粮食及农业组织 (FAO)把《公约》的目标摆在突出地位, 并纳入部门 计划。

\section{2 缔约方大会的作用}

《公约》的缔约方大会是《公约》的议事和决 策机制, 负责审查《公约》的实施情况，具有通过 议定书和相关决议、建立有关附属机构等功能，推 进、监督和引导着《公约》的发展和走向(CBD, 1992), 可以说是《公约》的最高权力机构，也是《公约》 履约机制在国际层面的主要构成部分(张丽荣等, 2009)。经过最初几年的调整，缔约方大会逐渐固定 为每两年举行一次, 会期约两周, 同期举行 《公约》 下属两个议定书的缔约方会议。按照惯例，期间东 道国还将主办环境部长高级别会议。缔约方大会迄 今已举办过 13 次, 5 个亚洲国家曾任大会东道国, 分 别是印度尼西亚(COP2)、马来西亚(COP7)、日本 (COP10)、印度(COP11)和韩国(COP12)。

COP15将于2020年召开。“联合国生物多样性十 年”将于 2020 年结束, COP15大会将评估《生物多样 性战略计划》(2011-2020)实施进展。如能达成协议, 将出台2020后全球保护生物多样性目标, 为下一个 
10年甚至更长时期的全球生物多样性治理确立基本 框架, 其意义与影响不言而喻。由此可见, COP15 将在《公约》进程中占据极为特殊的历史地位。

\section{2 举办缔约方大会的成本与驱动力}

\section{1 举办缔约方大会的多方位投入}

举办缔约方大会需要东道国付出可观的经济 与政治投入。其一, 缔约方大会由各缔约方派出代 表团参与, 相关的国际组织、非政府组织、土著和 地方社区也可派代表作为观察员出席(SCBD, 2001), 其级别高、涉及面广泛、参会人员众多, 资金、人 力和物力等方面所需投入不菲(表1)。除了会议本身 的组织外, 缔约方大会的成功与否还依赖于东道国 做出的特殊贡献。德国在COP9上提出了 “生命网络 倡议” (LifeWeb Initiative), 2008-2010年先后投入8 亿欧元支持了 30 余个子项目的实施。德国还出资支 持《公约》下有关专家会议的举行, 并承诺在 2010-2012年间出资至少3.5亿欧元支持“减少毁林 和森林退化所致排放量计划” (REDD), 增强《公约》 与气候变化之间的联系。日本在COP10上提出的“和 谐生命倡议” (Life in Harmony Initiative)计划出资 20 亿美元, 支持发展中国家开展生物多样性保护活 动。COP10上, 关于《名古屋议定书》文本的谈判 一度陷入僵局, 在最后时刻, 日本不得不提出了主 席国提案，并承诺出资10亿日元建立“日本生物多 样性基金”, 另外再增资 10 亿日元帮助发展中国家 进行能力建设, 促成大会最终通过了《名古屋议定 书》。韩国为实现其在COP12上提出的“平昌路线图” (Pyeongchang Roadmap), 推出了五项倡议, 涉及森
林、海洋、生物安全、跨境保护区等领域, 通过提供 资金与人员支持, 开展了系列能力建设与试点项目。

其二, 缔约方大会是一个连续性的多边进程, 经过13次大会和诸多附属机构会议，形成了众多的 谈判议题与复杂的国家集团分化(张丽荣等, 2009)。 从前期成果文件的准备, 到会议的主持和谈判, 需 要在不同的场合开展大量外交协调工作。通常, 东 道国的部长级官员将出任大会主席, 主持全会等重 要会议, 并每天召开主席团会, 促成谈判达成一 致。此外, 东道国还需为大会的顺利进行做好前期 铺垫和后期衔接工作, 如负责主持在下次缔约方会 议召开之前的主要工作组、附属执行机构等会议, 并与前后任主席国做好交接。以上活动均对东道国 的政治意愿、议程设定能力和谈判把控能力提出了 较高要求。

\section{2 举办缔约方大会的内在动因}

国家不惜气力举办这样一次缔约方大会的动 因, 来自于举办缔约方大会国家内政和外交核心 利益的深度契合。多数东道国会努力使会议成果 能够在一定程度上反映本国的关注和理念。以德 国为例，作为欧盟的领头羊，其环境外交背靠欧盟 国家在环境领域的政治意愿、强大财力和环境科技 实力, 是全球多边环境外交舞台上最为重要的规则 制定者和推手之一(张海滨, 2008)。在担任主席国期 间，德国无论从议程设置、资金投入还是外交斡旋 上，都展现了领导者的姿态。

日本同样将环境外交放在本国外交的主流地 位。对日本而言, 环境保护是以和平国家形象对世 界作出贡献的最合适的领域(林晓光, 1994), 日本在

表1 近5次缔约方大会(COP)基本情况

Table 1 Basic information on five recent Conference of Parties (COP)

\begin{tabular}{|c|c|c|c|c|c|}
\hline $\begin{array}{l}\text { 会议 } \\
\text { COP }\end{array}$ & $\begin{array}{l}\text { 时间 } \\
\text { Time }\end{array}$ & $\begin{array}{l}\text { 地点 } \\
\text { Venue }\end{array}$ & $\begin{array}{l}\text { 注册人数 } \\
\text { No. of delegates }\end{array}$ & $\begin{array}{l}\text { 东道国出席领导人 } \\
\text { Attending leader of the host country }\end{array}$ & $\begin{array}{l}\text { 来源 } \\
\text { Source }\end{array}$ \\
\hline COP9 & $\begin{array}{l}\text { 2008年5月19-30日 } \\
\text { May 19-30, 2008 }\end{array}$ & $\begin{array}{l}\text { 德国波恩 } \\
\text { Bonn, Germany }\end{array}$ & 7,000 & 总理 Chancellor & Evans \& Davies, 2014 \\
\hline COP10 & $\begin{array}{l}\text { 2010年10月18-29日 } \\
\text { October 18-29, } 2010\end{array}$ & $\begin{array}{l}\text { 日本名古屋 } \\
\text { Nagoya, Japan }\end{array}$ & 7,000 & 首相 Prime Minister & Evans \& Davies, 2014 \\
\hline COP11 & $\begin{array}{l}\text { 2012年10月 8-19日 } \\
\text { October 8-19, } 2012\end{array}$ & $\begin{array}{l}\text { 印度哈德拉巴德 } \\
\text { Hyderabad, India }\end{array}$ & 6,000 & 总理 Prime Minister & Evans \& Davies, 2014 \\
\hline COP12 & $\begin{array}{l}\text { 2014年10月6-17日 } \\
\text { October 6-17, 2014 }\end{array}$ & $\begin{array}{l}\text { 韩国平昌 } \\
\text { Pyeongchang, Republic of Korea }\end{array}$ & 3,000 & 总理 Prime Minister & IISD, 2014 \\
\hline COP13 & $\begin{array}{l}\text { 2016年12月2-17日 } \\
\text { December 2-17, } 2016\end{array}$ & $\begin{array}{l}\text { 墨西哥坎昆 } \\
\text { Cancun, Mexico }\end{array}$ & 8,000 & 总统 President & IISD, 2016 \\
\hline
\end{tabular}


$\mathrm{COP} 10$ 的举办上可谓全民动员，投入了巨大的精 力。一方面, 利用一切可能的双多边渠道进行对外 沟通, 包括联合国大会、G8峰会和高层出访, 提高 国际关注, 推动达成共识; 另一方面, 也非常注重 对其所作工作的宣传展示, 力求建立 “大国形象” (The Government of Japan, 2014), 占领国际环境外 交主导权。

韩国作为东北亚的一个地区性强国, 举办大会 的目标是在全球生物多样性议程中有所贡献, 同时 借此机会在韩国国内推动生物多样性保护, 提升国 际形象。以此为出发点, 韩国在会议的组织和成果 的推动上也作出了与之相称的努力, 提出了包含推 动战略计划实施一揽子措施的平昌路线图, 在联合 国框架下的多次政府间协商会议中发挥着主导作 用，推动了生物多样性目标被纳入联合国的可持续 发展目标(SDG) (The Government of the Republic of Korea, 2016)。

\section{3 缔约方大会带来的政治收益}

成功举办的缔约方大会不仅服务于国家利益, 也为东道国带来了直接的政治收益。主要体现在体 系话语权的提升、国际形象的塑造和国内环境管理 制度的优化三个方面。

首先, 就话语权和主动权的获得而言, 从历次 大会所取得的成果可以看出, 几次成果较为重要的 缔约方大会中, 东道国均发挥了强有力的议程设定 能力, 并产生了持续的影响(表2)。最典型的例子是 日本举办的 COP 10 。会上通过的战略计划中 5 个战 略目标及 20 个纲要目标被统称为“爱知生物多样性 目标”, 在长达 10 年的时间里, 生物多样性的目标 都与 “爱知”二字联系在一起, 为各国履约提供了责 任坐标与政策导向。德国在担任主席国期间提出了 GOBI (全球海洋生物多样性倡议)、TEEB (生物多 样性和生态系统经济学)研究倡议等, 在此后 10 余 年的谈判和履约进程中占据了重要位置。

其次, 大会为东道国带来的形象加分是显而易 见的。国家形象与定位作为软实力的组成要素, 同 样是主导国际规则能力的重要来源(Nye, 1990)。印 度于 2012 年承办的第11次缔约方大会, 虽未留下与 《波恩准则》、《名古屋议定书》同等标志性的成果, 但作为东道国, 印度在会上播出、发放了大量的生 物多样性宣传材料, 给与会代表留下了印度作为 “生物多样性丰富国家”和“生物多样性保护大国”的
深刻印象。COP10对日本环境外交大国形象的塑造 更是成功的。《公约》秘书处和多国都曾在不同场 合称赞日本对生物多样性领域国际合作的贡献，前 《公约》执秘艾哈迈德 - 朱格拉夫曾表示: “我很赞 赏日本。尽管日本在经历一场通缩危机……但他们 通过设立日本生物多样性基金表明了一种政治立 场” (Gupta, 2010)。

最后，大会的筹办过程能够从决策和施政等多 个方面优化东道国国内的环境管理制度。表现在生 物多样性主流化程度大大提升, 在政府治理中得到 充分考量, 不同部门甚至区域间的生物多样性政策 协同实施得到增强，社会公众对于国内相关环境治 理的参与度显著提高，相关政策的实施得到推动。 在 COP9举办前，欧洲政府与民间发起的“倒数 2010” (Countdown 2010)活动形成了浩大声势, 为 提高社会公众对生物多样性损失的认识、加强国家 间协作起到了积极作用(柴立伟等, 2015)。调查显示, 日本民众对生物多样性的认识在缔约方大会后出 现显著提升, 此外, 社会各界力量深度参与了 COP10的准备(MOEJ, 2010), 充分发挥了企业等利 益做关方对国内生态环境治理的积极性。为更好地 支持平昌路线图的实施，韩国国内加强了相关部委 的协同，环境部、森林服务局、海洋事务与渔业部 以及贸易、工业与能源部共同筹备大会, 提出各有 侧重但互相关联的倡议(The Government of the Republic of Korea, 2016)。

\section{3 举办COP15的“中国方案”}

展望2020, COP15由于其特殊意义而为世界所 关注。对于东道国而言, 这既蕴藏着机遇, 也是巨 大挑战。况且COP15的特殊意义不仅仅存在于国际 层面，对国内而言，其潜在影响同样重大而深远： 2020年是中国“十三五”的收官之年，也是全面建成 小康社会目标的达成之年, 届时国家生态文明体系 将得以全面构建。本届政府将生态文明作为国家战 略提升到前所未有的高度, 习近平总书记和李克强 总理多次就生物多样性保护作出重要批示。生物多 样性不仅是生态文明建设的应有之义， 《公约》下 多项热点议题更与我国核心利益息息相关。可以判 断, 在此承前启后的重要时间节点举办COP15, 符 合中央生态文明建设和参与全球治理的思路与要 求，是落实生态文明体制改革的有力抓手，同时也 
表2 历届缔约方大会主要成果一览表

Table 2 Major outcomes of Conference of the Parties (COP) to the Convention on Biological Diversity (CBD)

\begin{tabular}{|c|c|c|c|}
\hline $\begin{array}{l}\text { 年份 } \\
\text { Year }\end{array}$ & $\begin{array}{l}\text { 会议 } \\
\mathrm{COP}\end{array}$ & $\begin{array}{l}\text { 主办国 } \\
\text { Host country }\end{array}$ & $\begin{array}{l}\text { 主要成果 } \\
\text { Major outcomes }\end{array}$ \\
\hline 1994 & COP1 & $\begin{array}{l}\text { 巴哈马 } \\
\text { Bahamas }\end{array}$ & $\begin{array}{l}\text { 通过公约执行框架、缔约方大会中期工作方案(1995-1997年); 建立科学技术合作信息交换所机制 } \\
\text { (CHM)与科学、技术和工艺咨询附属机构(SBSTTA); 确定全球环境基金(GEF)为《公约》的资金机制 } \\
\text { Adopt the framework for the implementition of the CBD and the medium-term programme of work of the } \\
\text { Conference of the Parties (1995-1997); Establish the Clearing House Mechanism (CHM) and the Subsidi- } \\
\text { ary Body on Scientific, Technical and Technological Advice (SBSTTA); Decide the Global Environment } \\
\text { Facility (GEF) as financial mechanism }\end{array}$ \\
\hline 1995 & COP2 & $\begin{array}{l}\text { 印度尼西亚 } \\
\text { Indonesia }\end{array}$ & $\begin{array}{l}\text { 通过关于海洋及沿海生物多样性的《雅加达任务》; 成立生物安全临时工作组, 开始生物安全议定书 } \\
\text { 的谈判 } \\
\text { Adopt Jakarta Mandate on Marine and Costal Biodiversity; Establish the Ad-hoc Working Group on Bio- } \\
\text { safety to start the negotiations on biosafety protocol }\end{array}$ \\
\hline
\end{tabular}

1996 COP3 阿根廷

Argentina

通过《公约》与GEF的《谅解备忘录》; 开始农业生物多样性、森林生物多样性、生态系统方法和8(j) 条款的讨论

Adopt the Memorandum of Understanding with GEF; Start discussions on agricultural biodiversity, forest biodiversity, ecosystem approach and Article 8(j)

1998 COP4 斯洛伐克

Slovakia

2000

COP5

成立 $8(\mathrm{j})$ 条款不限名额特设工作组、ABS专家组; 通过海洋与沿海生物多样性、森林生物多样性和内 陆水域生物多样性工作方案、全球生物分类学倡议(GTI)

Establish an Ad-hoc Working Group on Article 8(j) and a Panel of Experts on ABS; Adopt the Programme of Work on marine and coastal biodiversity, forest biodiversity and inland biodiversity, and the Global Taxonomy Initiative (GTI)

\begin{tabular}{|c|c|c|c|}
\hline & & & $\begin{array}{l}\text { 工作方案; 《公约》与《拉姆萨湿地公约》的联合工作计划; 生态系统方法准则 } \\
\text { Cartagena Protocol on Biosafety opens for signature; Adopt Programmes of Work on dry and sub-humid } \\
\text { lands and agriculture biodiversity, the Joint Work Plan of the Convention on Biological Diversity and the } \\
\text { Convention on Wetlands (Ramsar, Iran, 1971) and the Ecosystem Approach Principles }\end{array}$ \\
\hline 2002 & COP6 & $\begin{array}{l}\text { 荷兰 } \\
\text { the Netherlands }\end{array}$ & $\begin{array}{l}\text { 通过《公约》战略计划(2002-2010年)、波恩准则、全球生物分类学倡议工作方案、全球植物保护战略 } \\
\text { Adopt the Strategic Plan (2002-2010), the Bonn Guidelines on ABS, the Programme of work on Global } \\
\text { Taxonomy and the Global Strategy for Plant Conservation }\end{array}$ \\
\hline 2004 & COP7 & $\begin{array}{l}\text { 马来西亚 } \\
\text { Malaysia }\end{array}$ & $\begin{array}{l}\text { 通过山地生物多样性、保护区、技术转让与合作工作方案; 成立岛屿生物多样性特设专家组; 授权 } \\
\text { ABS工作组开始进行ABS机制设计谈判 } \\
\text { Adopt Programme of Work on mountain biodiversity, protected areas and technology transfer; Establish } \\
\text { Ad-hoc Technical Expert Group on island biodiversity; Give Ad-hoc Working Group on ABS the mandate } \\
\text { to start negotiations on the ABS regime design }\end{array}$ \\
\hline 2006 & COP8 & 巴西 Brazil & $\begin{array}{l}\text { 通过岛屿生物多样性工作方案; 成立遗传资源起源地、来源和合法出处证书特设技术专家组 } \\
\text { Adopt the Programme of Work on island biodiversity; Establish a group of technical experts on an interna- } \\
\text { tionally recognized certificate of origin/source/legal provenance }\end{array}$ \\
\hline 2008 & COP9 & 德国 Germany & $\begin{array}{l}\text { 成立生物多样性与气候变化特设技术专家组; 通过ABS国际机制谈判路线图 } \\
\text { Establish the Ad-hoc Technical Expert Group on Biodiversity and Climate Change; Adopt a roadmap for } \\
\text { the negotiation of international ABS regime }\end{array}$ \\
\hline 2010 & COP10 & 日本 Japan & $\begin{array}{l}\text { 通过《名古屋议定书》、全球生物多样性战略计划(2011-2020年)、资源调动战略执行框架 } \\
\text { Adopt the Nagoya Protocol, Strategic Plan for Biodiversity 2011-2020, and the Framework for the Imple- } \\
\text { mentation of the Resource Mobilization Strategy }\end{array}$ \\
\hline 2012 & COP11 & 印度 India & $\begin{array}{l}\text { 提出到2015年流向发展中国家的生物多样性相关国际财政资源翻一番，并至少维持到2020年的初步 } \\
\text { 目标 } \\
\text { Put forward a preliminary target of doubling the financial resource flows to developing countries by } 2015 \\
\text { and maintaining the momentum to at least } 2020\end{array}$ \\
\hline 2014 & COP12 & $\begin{array}{l}\text { 韩国 } \\
\text { Republic of Korea }\end{array}$ & $\begin{array}{l}\text { 形成平昌路线图; 设立执行问题附属机构 } \\
\text { Adopt the Pyeonchang Roadmap; Create the Subsidiary Body on Implementation }\end{array}$ \\
\hline 2016 & COP13 & 墨西哥 Mexico & $\begin{array}{l}\text { 提出《坎昆宣言》, 促进生物多样性主流化; 通过生态系统恢复短期行动计划 } \\
\text { Put forward the Cancun declaration on mainstreaming; Adopt a short-term action plan on ecosystem resto- } \\
\text { ration }\end{array}$ \\
\hline
\end{tabular}

是服务国家外交战略, 讲述中国故事, 与国际社会 共谋全球生态文明建设之路的重大机遇。

同时应当认识到, 举办此次大会将对我国在生
物多样性领域把握议题和谈判斡旋的能力提出挑 战。国际社会期待COP15能够达成2020后全球保护 生物多样性目标, 东道国将肩负繁重的外交协调任 
务, 或许需要为保障会议成果的达成作出实质性政 治许诺。我国虽然是最早加入《公约》的国家之一, 但25年来尚未承担过其框架下的全球性会议。我国 的履约工作呈现出显著的内向型特征, 尤其表现在 对外合作以“以外促内”为重点, 争取国际资金的 “输入”来推动国内生物多样性保护和生物资源可持 续利用，对所在区域或全球的资金或技术“输出”尚 且非常有限; 以及在国际谈判中 “不出头不扛旗”, 被动参与涉及本国和发展中国家权益的议题, 较少 主动设置议程或引领谈判进程。承办COP15将要求 我国生物多样性领域的国际合作实现从内向型到 外向型的过渡, 如此才能在未来 10 年甚至更为长远 的国际规则制定进程中, 切实维护我国正当权益, 真正树立环境大国的形象。

基于以上考量, 我们认为在大会的筹办中当在 以下方面做出努力:

(1)在会议筹备上, 强化顶层设计, 扭转过去我 国在多边进程中的“被动应对”的局面, 寻求更大的 战略主动。早做筹谋, 充分发挥中国生物多样性保 护国家委员会的作用, 统筹各相关部委, 发动社会 各界力量; 重视科研基础, 立足于国际科研前沿, 引入世界先进水平的智囊团参与成果文件设计; 建 立由多国优秀人才组成的大会筹备团队, 以国际化 的视野与胸襟运用好东道国的主场优势; 将大会筹 备列入国家外交主流日程, 充分利用联合国、区域 合作、首脑外交、部长外交等各种双、多边外交场 合提高大会的政治关注度, 协调各方立场, 为大会 打下良好基础。

(2)在谈判立场上, 通过战略协同, 维护中国和 发展中国家的整体利益。做好周边外交, 利用中日 韩、中国-东盟等机制与邻国提前沟通, 存异而求 同。为《公约》秘书处引领的相关国际规则制定进 程, 特别是生物多样性国际体系长远制度的构建提 供有力支持。坚持紧紧围绕《公约》的3大目标, 以 生态系统管理的科学方法为基础, 避免 《公约》谈 判重心偏离到较为外缘、敏感的新议题上去(柴立伟 等, 2015)。

(3)在东道国贡献上, 争取达成两点。一要为国 际履约提供范式选择和经验参考。中国的生物多样 性工作具有鲜明特色, 其行政管理范式区别于西方 传统的经济驱动和公民社会驱动手段, 切合自身发 展阶段和国内实际，在自然保护地、生态红线、转
移支付、生态扶贫等制度性安排方面取得了显著的 成效。应注重继续抓好抓实国内生物多样性工作, 带动各部门和地方积极总结成功经验，在大会上以 主题边会、展览、资料发放等多样化的形式，展现 我国生态文明建设的生动实践。二要制定与国家外 交战略和综合国力相称的参与策略, 尽早启动相关 可行性研究, 协助新一轮全球生物多样性保护目标 与路线图的设计与落实。

自此, 中国将能够通过筹办大会开辟新的战略 空间，实现对全球环境治理的深度参与。随着中国 在全球生态环保事业中的参与度和话语权的提升, 环境外交也将进一步走向战略前台, 为国家大外交 的转型提供强大助力。

\section{参考文献}

CBD (1992) Text of the Convention. https://www.cbd.int/convention/text/. (accessed on 2017-07-10)

Chai LW, Cao XF, Zhang JQ, Huang Y (2015) Trends of the implementation of "Convention on Biological Diversity" after development of "Aichi Targets” and countermeasures. Journal of Ecology and Rural Environment, 31(1), 7-11. (in Chinese with English abstract) [柴立伟, 曹晓峰, 张洁清, 黄艺 (2015) “爱知目标” 后 《生物多样性公约》履约趋势 分析和对策. 生态与农村环境学报, 31(1), 7-11.]

Evans JW, Davies R (2014) Too Global to Fail: The World Bank at the Intersection of National and Global Public Policy in 2025. World Bank Publications, Washington, DC.

Gupta J (2010) Stepping up the fight for life (2). https://www. chinadialogue.net/article/show/single/ch/3859-Stepping-upthe-fight-for-life-2-/. (accessed on 2017-08-07)

IISD Reporting Services (IISD) (2014) Earth Negotiations Bulletin. http://enb.iisd.org/biodiv/cop12/. (accessed on 201709-16)

IISD Reporting Services (IISD) (2016) Earth Negotiations Bulletin. http://enb.iisd.org/download/pdf/enb09678e.pdf/. (accessed on 2017-08-10)

Li BJ, Xu ZY (2006) China's self-identity construction as a responsible power in the post-cold war era. Teaching and Research, (1), 49-56. (in Chinese with English abstract) [李 宝俊, 徐正源 (2006) 冷战后中国负责任大国身份的建 构. 教育与研究, (1), 49-56.]

Lin XG (1994) Environmental diplomacy of the government of Japan. Japanese Studies, (1), 19-32. (in Chinese) [林晓光 (1994) 日本政府的环境外交. 日本学刊, (1), 19-32.]

Ministry of Environment of Japan (MOEJ) (2010) Annual Report on the Environment, the Sound Material-Cycle Society and Biodiversity in Japan 2010. https://www.env.go.jp/en/ wpaper/2010/index.html/. (accessed on 2017-07-20)

Nye JS (1990) Bound to Lead: The Changing Nature of Amer- 
ican Power. Basic Books Inc., New York.

Qin YQ (2003) National identity, strategic culture, and security interests: three hypotheses on the relationship between China and international society. World Economics and Politics, (1), 10-15. (in Chinese with English abstract) [秦亚青 (2003) 国家身份、战略文化和安全利益——关于中国与 国际社会关系的三个假设. 世界经济与政治, (1), 10-15.]

Secretariat of the Convention on Biological Diversity (SCBD) (2001) Handbook of the Convention on Biological Diversity Including Its Cartagena Protocol on Biosafety. Earthscan, London.

Shi YH (2011) Economy, security and ecology - three major global challenges and China. China Report, (12), 26-29. (in Chinese) [时殷弘 (2011) 经济、安全、生态: 三大全球性 挑战与中国. 中国报道, (12), 26-29.]

The Government of Japan (2014) Report on Activities of Japan as President and the Host Country of COP10. https://www. cbd.int/cop/cop-presidency/cop-10-presidency-report-en.pdf/. (accessed on 2017-07-22)

The Government of the Republic of Korea (2016) Report on Korea's CBD Presidency of COP12. https://www.cbd.int/ cop/cop-presidency/cop-12-presidency-report-en.pdf/. (accessed on 2017-08-03)

United Nations (UN) (2015) Sustainable development. https: //sustainabledevelopment.un.org/. (accessed on 2017-08-15)

Yu HY (2013) International Climate Environment Diplomacy: China's Responses. Orient Publishing Center, Shanghai. (in Chinese) [于宏源 (2013) 国际气候环境外交: 中国的应 对. 东方出版中心, 上海.]

Zhang HB (2008) Environment and International Relations: Rational Thinking on Global Environmental Issues. Shanghai People's Publishing House, Shanghai. (in Chinese) [张 海滨 (2008) 环境与国际关系: 全球环境问题的理性思 考. 上海人民出版社, 上海.]

Zhang LR, Cheng WJ, Xue DY (2009) Progress and trends for implementation of the convention on biological diversity. Acta Ecologica Sinica, 29, 5636-5643. (in Chinese with English abstract) [张丽荣, 程文娟, 薛达元 (2009) 《生物 多样性公约》国际履约的进展与趋势. 生态学报, 29 , 5636-5643.]

(责任编委: 薛达元 责任编辑: 黄祥忠) 